\title{
Studies of 4-rotor unmanned aerial vehicle UAV in the field of control system
}

\author{
Lucjan Setlak $^{1 *}$, Rafat Kowalik ${ }^{1 *}$ \\ ${ }^{1}$ Department of Avionics and Control Systems, Polish Air Force Academy, ul. Dywizjonu 303 nr 35, 08-521 Deblin
}

\begin{abstract}
The key goal of this work was to develop a functional mathematical model of a 4-rotor UAV, including regulatory apparatus and identification of its parameters. The functionality of a quadrocopter traffic control has been reduced to solving differential equations that define the motion and dynamics of an unmanned aerial vehicle. It should be noted that the synthesis of the quadrocopter control system is not an easy task, due to the non-linear nature of the dynamics of this object and its structural instability. Therefore, in this article the tested object UAV was accepted as a physical model, which may cause potential material damage resulting from damage to the device as well as other elements that are located in its immediate surroundings. In addition, the article discusses the problem of improving the quality of the estimation rate of climb of unmanned aircraft of vertical takeoff and landing UAV, this problem was considered for the object in the low-ceiling range, i.e. in the range of 0-6 m, so the issue concerns autonomous take-off and landing. For the presentation of the results, the 4-rotor UAV was used, with the use of a proportional-integralderivative PID controller in the context of the control system. The obtained results were supported by research and analysis of real results - the discussed algorithm was implemented in the 4-rotor UAV driver.
\end{abstract}

\section{Introduction}

When reviewing and making a preliminary analysis of the literature in the scope of the subject of the research it should be noted that currently there are many developed methods of controlling Unmanned Aerial Vehicles (UAVs). Such methods include: classic control, linearquadratic regulator, backstepping method, sliding mode control, non-linear control and reinforcement learning.

Classic control - the controller consists of three parts: proportional, integral and differential. The proportional member is responsible for the basic feedback, integral removes the determined states, and differential adds damping to the controller. Research has shown that this type of control makes it possible to perform hovering, but it does not work in terms of four-rotor UAV control during severe air disturbances. In addition, when flying at low speeds with small aerodynamic disturbances, classic control is sufficient to maintain full control of the UAV. When flying at higher speeds, the multi-rotor UAV becomes very sensitive to external factors, so other control methods must be used [1], [2], [3].

Linear-quadratic regulator - this type of control is characterized by the independent use of the Riccati equation. During the tests, it was noticed that the tilting and inclination of the multi-rotor UAV significantly oscillates, making the aircraft not able to make a hover. Despite many attempts, it was not possible to achieve a satisfactory result. Continuous work (activity) - as the results of research on the control method using a linearquadratic controller proved to be insufficient, the focus was on a different type of control. This technique can rapidly stabilize the chain of integrators with the limit entry. Preliminary experiments have shown that such cheap systems are classified at a satisfactory level.

Backstepping method - this type of control principle is designed based on the Lyapunov stability criterion. The main idea of this project is to step back through the system to provide control. It is particularly useful when certain states are controlled by other states, as is the case with the dynamics of the four-rotor UAV.

Sliding Mode Control - this is a type of control using non-linear systems, in which the dynamics of a nonlinear system is modified by using a discontinuous control signal. The variables of the feedback loop state in this case are not a function of time.

Non-linear control - control is carried out by converting a known non-linear system to the corresponding linear one, by changing the components and the corresponding control input. A specific nonlinear control type is dynamic non-linear inversion. The non-linear model is presented as linear and inverted. The linear system created in this way is placed in the inner loop, and the outer loop is added to the inner loop control.

Reinforcement learning - in this case, the non-linear and non-parametric control model is initially constructed based on flight data, approximating the random Markov processes. Then, the appropriate algorithm searches for

\footnotetext{
*Corresponding author: 1.setlak@wsosp.pl, r.kowalik@wsosp.pl
} 
the appropriate control method that can be implemented on embedded microprocessors.

This type of control is sensitive to interferences that have not been previously stored in the system. In addition, special changes in the battery charge level and damage to the rotor blades can reduce the stability or steady state of the delay. After analyzing the key fourmotor control strategies of the UAV, it can be concluded that not all of them lead to similar results.

Comparing the advantages and disadvantages of the presented solutions, the classic control method proved to be the best choice. Despite the simplest design, it works well enough in application in a four-rotor UAV, used in a confined space, and therefore further considerations will be made on this basis [4], [5].

\section{Mathematical model of 4-rotor UAV in the field of control}

When approaching the mathematical description (model) of a multi-rotor UAV in terms of control, it should be noted that the development of a dynamic UAV model, including its simulation, is necessary first of all in the aspect of testing in the field of navigation and control in a closed room. The model of dynamic equations, describing the character and position of the 4-rotor $\mathrm{UAV}$, is a fundamentally immovable construction with six degrees of freedom and four entrances. The dynamic model of the 4-rotor UAV system has already been thoroughly examined, tested and compared to the actual flight test results, therefore, it is sufficiently reliable to be used as a basis for simulating the model, without taking into account the additional effects that have been investigated in other publications [6], [7], [8].

The modeling process was started from defining the reference system, with two systems being distinguished in this respect. One of them is the gravitational system (associated with the Earth), described with axes $0_{e} X_{e} Y_{e} Z_{e}$, allowing to determine the motion of the aircraft (not taking into account the rotation of the Earth). In turn, the second system is the structural system (associated with aircraft), described in the axes $0_{b} X_{b} Y_{b} Z_{b}$, allowing to determine (in connection with the gravitational system) the spatial position of the aircraft [9], [10], [11]. The above systems are presented in the figure below (Fig. 1).

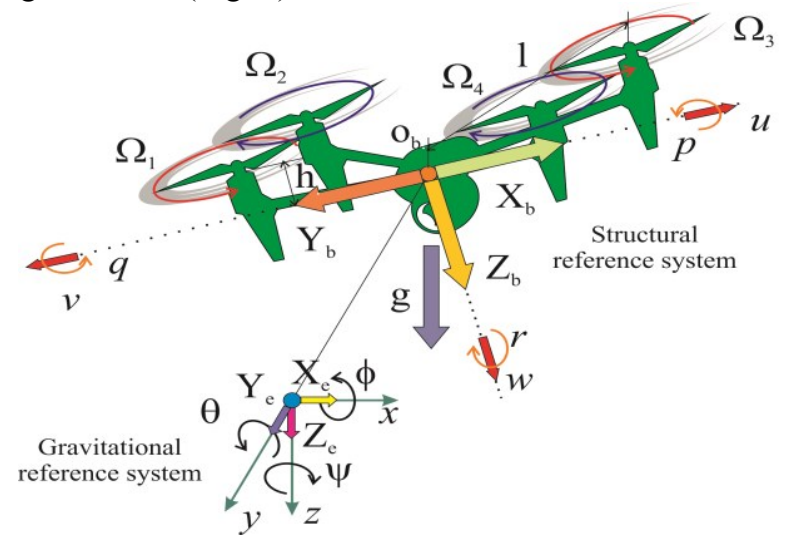

Fig. 1. Gravitational and structural reference system
The current position of the 4-rotor UAV is described by three axes $(x, y, z)$ with the center of gravity, taking into account the gravitational system. In turn, the current height is shown in the form of three Euler angles $(\psi, \theta$, $\phi)$. These three angles are respectively defined by a deviation $(-\pi \leq \psi<\pi)$, inclination $\left(-\frac{\pi}{2}<\theta<\frac{\pi}{2}\right)$ and tilting $\left(-\frac{\pi}{2}<\phi<\frac{\pi}{2}\right)$.

In the next stage of the modeling process was the presentation of kinematic links (relationships) relating to motion and rotation in the inertial reference system, connected with the Earth to the structural system. Derivatives with respect to time for angles $(\psi, \theta, \phi)$ can be expressed in the following form [12]:

$$
[\dot{\psi}, \dot{\theta}, \dot{\phi}]^{T}=N(\psi, \theta, \phi) \omega
$$

in which $\omega=[p, q, r]^{T}$ are angular velocities with reference to the reference construction system, and $N(\psi, \theta, \phi)$ is a matrix that can be represented in the following form:

$$
N(\psi, \theta, \phi)=\left[\begin{array}{ccc}
0 & \sin \phi \sec \theta & \cos \phi \sec \theta \\
0 & \cos \phi & -\sin \phi \\
1 & \sin \phi \tan \theta & \cos \phi \tan \theta
\end{array}\right]
$$

It should be noted that the matrix (2) depends only on $(\psi, \theta, \phi)$ and is reversible in case if its limits on $(\psi, \theta, \phi)$ are maintained. Analogously, a derivative with respect to the position time $(x, y, z)$ can be represented as:

$$
[\dot{\psi}, \dot{\theta}, \dot{\phi}]^{T}=N(\psi, \theta, \phi) \omega
$$

where: $V_{0}=\left[u_{0}, v_{0}, w_{0}\right]^{T}$ - is the current speed of the 4rotor UAV in relation to the reference system associated with the Earth. Determining by $V=[u, v, w]^{T}$ - the current UAV speed, expressed in the reference system associated with the aircraft, then $V$ and $V_{0}$ are bound by:

$$
V_{0}=R(\psi, \theta, \phi) \mathrm{V}
$$

where: $R(\psi, \theta, \phi)$ - describe the UAV rotation matrix:

$$
\begin{gathered}
\mathrm{R}(\psi, \theta, \phi)= \\
{\left[\begin{array}{ccc}
\cos \theta \cos \psi & (\sin \phi \sin \theta \cos \psi-\cos \phi \sin \psi) & (\cos \phi \sin \theta \cos \psi+\sin \phi \sin \psi) \\
\cos \theta \sin \psi & (\sin \phi \sin \theta \sin \psi+\cos \phi \cos \psi) & (\cos \phi \sin \theta \sin \psi-\sin \phi \cos \psi) \\
-\sin \theta & \sin \phi \cos \theta & \cos \phi \cos \theta
\end{array}\right]}
\end{gathered}
$$

For the purpose of creating a UAV model, fully compatible with reality, later in the article some simplifications have been made, among others: the UAV structure is rigid and symmetrical, the rotors are rigid, and the product of the inertia matrix and the Earth effect can be omitted [13], [14].

\subsection{Aerodynamic forces and moments acting on the rotor}

Using the theory of the blade element, it is possible to calculate the forces acting in parallel and perpendicular to the rotor shaft and the moments acting on the shaft and the rotor hub. Assuming that the rotors are rigid, forces acting parallel to the rotor shaft are defined as a 
rotor series $T$, while forces acting perpendicular to the rotor shaft act on the rotor hub $H$.

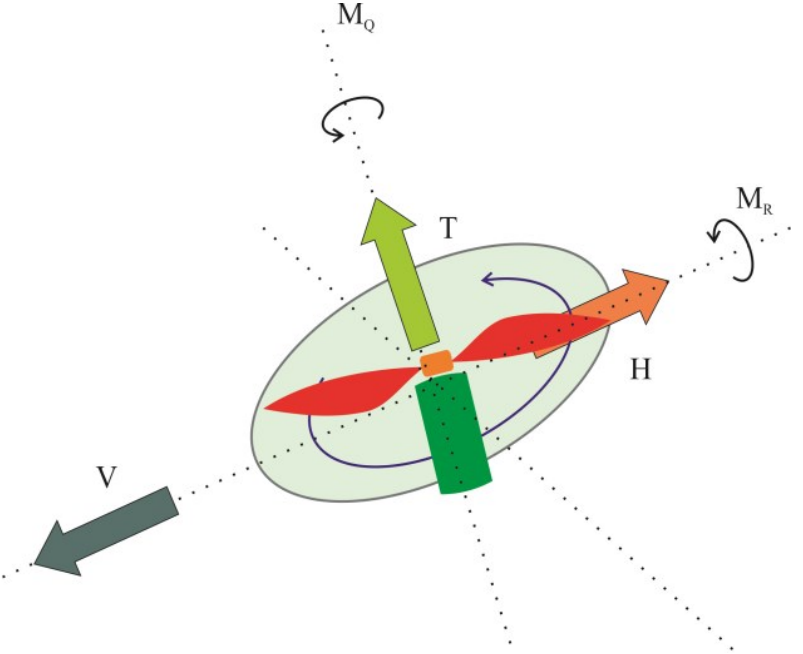

Fig. 2. Forces and moments affecting the equator

In addition, there are two moments acting on the rotor: moment of resistance $M_{Q}$ and torque $M_{R}$. It can be assumed that the lifting force acting on the rotor blade is an order of magnitude higher than the resistance. Correspondingly, forces and moments will be defined for each rotor. In the figure above (Fig. 2), forces and moments are illustrated.

Thrust results from the forces acting on all components of the blade perpendicular to the rotor shaft, which can be written [15], [16]:

$$
\begin{gathered}
T=C_{T \rho} A(\Omega R)^{2} \\
\frac{C_{T}}{\sigma a}=\left(\frac{1}{6}+\frac{\mu^{2}}{4}\right) \theta_{0}-\left(1+\mu^{2}\right) \frac{\theta_{t w}}{8}-\frac{\lambda}{4}
\end{gathered}
$$

In turn, the centrifugal force results from the forces acting on all blade elements in the horizontal plane, whereas the force being zero when the velocity is zero.

$$
\begin{gathered}
H=C_{H} \rho A(\Omega R)^{2} \\
\frac{C_{H}}{\sigma a}=\frac{\mu \bar{C}_{d}}{4 a}+\frac{1}{4} \lambda \mu\left(\theta_{0}-\frac{1}{2} \theta_{t w}\right)
\end{gathered}
$$

The moment of resistance results from all the forces acting on the center of the rotor in the horizontal plane, it determines the forces needed to maintain the rotation of the rotor:

$$
\begin{gathered}
M_{Q}=C_{M_{Q}} \rho A(\Omega R)^{2} \\
\frac{C_{M_{Q}}}{\sigma a}=\frac{1}{8 a}\left(1+\mu^{2}\right) \bar{C}_{d}+\lambda\left(\frac{\theta_{0}}{6}-\frac{\theta_{t w}}{8}-\frac{\lambda}{4}\right)
\end{gathered}
$$

When considering the tilting moment, it should be noted that due to the fact that the blades are moving in the horizontal plane in the air, the leading blade will produce more lift than returning, which affects the total torque generated by the rotor, which can be written as:

$$
M_{R}=C_{M_{R}} \rho A(\Omega R)^{2} R
$$

$$
\frac{C_{M_{R}}}{\sigma a}=\mu\left(\frac{\theta_{0}}{6}-\frac{\theta_{t w}}{8}-\frac{\lambda}{8}\right)
$$

\subsection{Dynamic equations}

Consider dynamical equations in the 4-rotor UAV range, initially it was assumed that the vector product of tensor of the moment of inertia $J$ can be omitted in relation to its structure [17], [18].

$$
J=\left[\begin{array}{ccc}
I_{x x} & 0 & 0 \\
0 & I_{y y} & 0 \\
0 & 0 & I_{z z}
\end{array}\right]
$$

Using the general equations of motion (9), written in the following form:

$$
\begin{gathered}
F_{x}=-W \sin \theta+X=m(\dot{u}+q w-r v) \\
F_{y}=W \cos \theta \cos \psi+Y=m(\dot{u}+r u-p w) \\
F_{Z}=W \cos \theta \cos \psi+Z=m(\dot{w}+p v-q u)
\end{gathered}
$$

and

$$
\begin{aligned}
& M_{X}=L=I_{x x} \dot{p}+\left(I_{z z}-I_{y y}\right) q r \\
& M_{Y}=M=I_{y y} \dot{q}+\left(I_{x x}-I_{z z}\right) r p \\
& M_{z}=N=I_{z z} \dot{r}+\left(I_{y y}-I_{x x}\right) p q
\end{aligned}
$$

where: $F_{x}, F_{y}, F_{z^{-}}$are external forces acting on the frame and $M_{x}, M_{y}, M_{z^{-}}$are external moments acting on the frame of the 4-rotor UAV.

In the further part of the paper, external forces and moments acting on the 4-rotor UAV arm were determined.

\subsubsection{External forces acting on the arm of 4-rotor UAV}

Forces acting along the axis $u$

Centrifugal force

Friction

Forces acting along the axis $v$

Centrifugal force

Friction

Forces acting along the axis $w$

Thrust

Friction

$$
\begin{aligned}
& -\sum_{i=1}^{4} H_{u_{i}} \\
& -\frac{1}{2} C_{u} A_{u} \rho u|u|
\end{aligned}
$$$$
-\sum_{i=1}^{4} H_{v_{i}}
$$$$
-\frac{1}{2} C_{v} A_{v} \rho v|v|
$$

$-\sum_{i=1}^{4} T_{i}$ $-\frac{1}{2} C_{w} A_{w} \rho w|w|$

\subsubsection{Moments acting on the arm of 4-rotor UAV}

Moments of tilting

Gyroscope effect of the propeller $\quad J_{r} q \Omega_{r}$

The tilt angle of the servomechanism $l\left(-T_{2}+T_{4}\right)$

The power of inertia caused by

traversing flight

$-h \sum_{i=1}^{4} H_{v_{i}}$ 
The torque caused by the forward flight

Inclining moments

Gyroscope effect of the propeller The tilt angle of the servomechanism The power of inertia caused by traversing flight

The inclining moment caused by the forward flight

Moments of deviation

Inertial moment of inertia

The moment of inertia during leading

out of balance

The forces acting on the hub in the forward flight during leading out of balance

The forces acting on the hub in the traverse flight during leading out of balance initially it was assumed that the vector product of tensor

\section{Moments of tilting}

Gyroscope effect of the propeller

The tilt angle of the servomechanism

The power of inertia caused by

traversing flight

The torque caused by the forward

flight

Inclining moments

Gyroscope effect of the propeller

The tilt angle of the servomechanism

The power of inertia caused by

traversing flight

The inclining moment caused by the

forward flight

Moments of deviation

Inertial moment of inertia

The moment of inertia during leading

out of balance

The forces acting on the hub in the forward flight during leading out of balance

The forces acting on the hub in the traverse flight during leading out of balance
$J_{r} q \Omega_{r}$

$(-1)^{i} \sum_{i=1}^{4} M_{R_{u_{i}}}$

$-J_{r} p \Omega_{r}$

$l\left(T_{1}-T_{3}\right)$

$h \sum_{i=1}^{4} H_{u_{i}}$

$(-1)^{i} \sum_{i=1}^{4} M_{R_{v_{i}}}$

$J_{r} \Delta \dot{\Omega}_{r}$

$(-1)^{i} \sum_{i=1}^{4} M_{Q_{i}}$

$l\left(H_{u_{2}}-H_{u_{4}}\right)$

$l\left(H_{v_{1}}-H_{v_{3}}\right)$

$l\left(-T_{2}+T_{4}\right)$

$-h \sum_{i=1}^{4} H_{v_{i}}$

$(-1)^{i} \sum_{i=1}^{4} M_{R_{u_{i}}}$

$-J_{r} p \Omega_{r}$

$l\left(T_{1}-T_{3}\right)$

$h \sum_{i=1}^{4} H_{u_{i}}$

$(-1)^{i} \sum_{i=1}^{4} M_{R_{v_{i}}}$

$J_{r} \Delta \dot{\Omega}_{r}$

$(-1)^{i} \sum_{i=1}^{4} M_{Q_{i}}$

$l\left(H_{u_{2}}-H_{u_{4}}\right)$

$l\left(H_{v_{1}}-H_{v_{3}}\right)$

\subsubsection{Full dynamic equations of 4-rotor UAV}

Combining the above equations we get:

$$
\begin{aligned}
m \dot{u}=-m(g \sin \theta & -q w+r v) \\
& -\sum_{i=1}^{4} H_{u_{i}}-\frac{1}{2} C_{u} A_{u} \rho u|u|
\end{aligned}
$$

$$
\begin{aligned}
m \dot{v}=m(g \cos \theta & \sin \phi-r u+p w) \\
& -\sum_{i=1}^{4} H_{v_{i}}-\frac{1}{2} C_{v} A_{v} \rho v|v|
\end{aligned}
$$

$$
m \dot{w}=m(g \cos \theta \sin \phi-p w+q u)
$$

$$
-\sum_{i=1}^{4} T_{i}-\frac{1}{2} C_{w} A_{w} \rho w|w|
$$

$$
\begin{array}{r}
I_{x x} \dot{p}=\left(I_{y y}-I_{z z}\right) q r+J_{r} q \Omega_{r}+l\left(-T_{2}+T_{4}\right) \\
-h \sum_{i=1}^{4} H_{v_{i}}+(-1)^{i} \sum_{i=1}^{4} M_{R_{u_{i}}}
\end{array}
$$

$$
I_{y y} \dot{q}=\left(I_{z z}-I_{x x}\right) r p+J_{r} p \Omega_{r}+l\left(T_{1}-T_{3}\right)
$$$$
+h \sum_{i=1}^{4} H_{u_{i}}+(-1)^{i} \sum_{i=1}^{4} M_{r_{v_{i}}}
$$

$$
\begin{aligned}
I_{z z} \dot{r}=\left(I_{x x}-I_{y y}\right) & p q+J_{r} \dot{\Omega}_{r}+(-1)^{i} \sum_{i=1}^{4} M_{Q_{i}} \\
& +l\left(H_{u_{2}}-H_{u_{4}}\right) \\
& +l\left(-H_{v_{1}}+H_{v_{3}}\right)
\end{aligned}
$$

The above dynamic equations (13) constitute a complete model of the 4-rotor UAV with the previously given assumptions. However, it should be noted that despite the negligible impact of several factors (e.g. the occurrence of the earth effect), after performing appropriate tests, they may be considered when designing the next models.

\subsection{Simplified model of the control system and filter design}

For the design of the control system and the filter, the use of a simplified model is sufficient, because it allows easier estimation of filter operation and control principles. The simplified model is as follows [19], [20]:

$$
\begin{gathered}
m \dot{u}=-m(g \sin \theta-q w+r v) \\
m \dot{v}=m(g \cos \theta \sin \phi-r u+p w) \\
m \dot{w}=m(g \cos \theta \cos \phi-p v+q u)-U_{1} \\
I_{x x} \dot{p}=\left(I_{y y}-I_{z z}\right) q r+l U_{2} \\
I_{y y} \dot{q}=\left(I_{z z}-I_{x x}\right) r p+l U_{3} \\
I_{z z} \dot{r}=\left(I_{x x}-I_{y y}\right) p q+l U_{4}
\end{gathered}
$$

where: $U_{1}, U_{2}, U_{3}, U_{4^{-}}$are the entries described in subsequent equations.

During hover, the thrust $T_{i}$ for each rotor is estimated as:

$$
T_{i} \approx b \Omega_{i}^{2}
$$

where: $b$ - is the thrust ratio in hover.

There is a similar relationship for torque $M_{Q_{i}}$ :

$$
M_{Q_{i}} \approx d \Omega_{i}^{2}
$$


where: $d$ - is the coefficient of resistance.

Therefore, in this case the entries can be described as [21], [22]:

$$
\begin{gathered}
U_{1}=\sum_{i=1}^{4} T_{i}=b\left(\Omega_{1}^{2}+\Omega_{2}^{2}+\Omega_{3}^{2}+\Omega_{4}^{2}\right) \\
U_{2}=\left(-T_{2}+T_{4}\right)=b\left(-\Omega_{2}^{2}-\Omega_{4}^{2}\right) \\
U_{3}=\left(T_{1}-T_{3}\right)=b\left(\Omega_{1}^{2}-\Omega_{3}^{2}\right) \\
U_{4}=(-1)^{i} \sum_{i=1}^{4} M_{Q_{i}}=d\left(-\Omega_{1}^{2}+\Omega_{2}^{2}-\Omega_{3}^{2}+\Omega_{4}^{2}\right)
\end{gathered}
$$

\subsection{Dynamic equations of the engine}

Modeling of engine operation can be based on the following equations [23], [24]:

$$
\begin{gathered}
\dot{\Omega}_{m}=-\frac{1}{\tau} \Omega_{m}-\frac{d}{\eta r^{3} J_{t}} \Omega_{m}^{2}+\frac{1}{k_{m} \tau} u \\
\frac{1}{\tau}=\frac{k_{m}^{2}}{R_{m} J_{t}}
\end{gathered}
$$

where: $d$ - is the drag coefficient during hovering, $\tau$ - is the engine time constant, $k_{m}$ - the torque constant, $R_{m}$ the internal resistance of the engine, $\eta$ - the engine efficiency and $u$ - is the voltage at the engine input.

Assuming the linearity of the rotor speed around the point $\Omega_{0}$, the engine rotational speed in hover can be represented as:

$$
\begin{gathered}
\dot{\Omega}_{m}=0=-\left(\frac{1}{\tau}+\frac{2 d \Omega_{0}}{\eta r^{3} J_{t}}\right) \Omega_{m}+\left(\frac{1}{k_{m} \tau}\right) u \\
+\frac{d \Omega_{0}^{2}}{\eta r^{3} J_{t}}
\end{gathered}
$$

The relationship between the required angular velocity and the engine voltage is described mathematically in the form:

$$
\mathrm{u}=\mathrm{k}_{\mathrm{m}} \tau\left(\left(\frac{1}{\tau}+\frac{2 \mathrm{~d} \Omega_{0}}{\eta \mathrm{r}^{3} \mathrm{~J}_{\mathrm{t}}}\right) \Omega_{\mathrm{m}}-\frac{\mathrm{d} \Omega_{0}^{2}}{\eta \mathrm{r}^{3} \mathrm{~J}_{\mathrm{t}}}\right)
$$

Then, using the previous equations, you can introduce simplifications:

$$
\begin{gathered}
u_{\text {thrust }}=\alpha_{\text {thrust }} \sqrt{T}+\beta \\
u_{\text {torque }}=\alpha_{\text {torque }} \sqrt{M_{Q}+\beta}
\end{gathered}
$$

In addition, the experiments carried out can be used to determine the relationship to calculate the voltage required by the torque and the thrust. By combining the previously given formulas, you can obtain a formula that allows you to calculate the output voltage of the engines:

$$
\begin{aligned}
& u=\left[\begin{array}{l}
u_{\text {silnik }_{1}} \\
u_{\text {silnik }_{2}} \\
u_{\text {silnik }_{3}} \\
u_{\text {silnik }_{4}}
\end{array}\right] \\
& \left.=k_{m} \tau\right)\left(\frac{1}{\tau}\right. \\
& \left.+\frac{2 d \Omega_{0}}{\eta r^{3} J_{t}}\right) \sqrt{\left[\begin{array}{cccc}
\frac{1}{4 b} & 0 & \frac{1}{2 b} & -\frac{1}{4 b} \\
\frac{1}{4 b} & -\frac{1}{2 b} & 0 & \frac{1}{4 b} \\
\frac{1}{4 b} & 0 & -\frac{1}{2 b} & -\frac{1}{4 b} \\
\frac{1}{4 b} & \frac{1}{2 b} & 0 & \frac{1}{4 b}
\end{array}\right]\left[\begin{array}{l}
U_{1} \\
U_{2} \\
U_{3} \\
U_{4}
\end{array}\right]} \\
& -\frac{d \Omega_{0}^{2}}{\eta r^{3} J_{t}}
\end{aligned}
$$

The above equation (22) is implemented to the block responsible for the operation of the engines during the simulation process in the Matlab/Simulink programming environment.

\subsection{Structure of 4-rotor UAV control}

As previously mentioned, the control mode chosen is a classic control, with the block reversing the engine operation added to the proposed control system.

\subsubsection{Deviation control}

Deviation is the least important channel that takes control into account, because it has no direct effect on the motion of the 4-rotor UAV. It can be independently tuned and tested, maintaining manual control in the other channels. Particular attention should be paid to the fact that different types of disturbances have a relatively small effect on the deviation, so no additional amplifiers are required to control the deviation direction.

All you need is a controller (driver) with low bandwidth connectors. The deviation control is described by the following formula [25], [26], [27]: 


$$
\begin{aligned}
U_{4}=-K_{\psi_{P}}(\psi- & \left.\psi_{d}\right) \\
& -K_{\psi_{I}} \int\left(\psi-\psi_{d}\right) \delta t \\
& -K_{\psi_{D}}\left(\dot{\psi}-\dot{\psi}_{d}\right)
\end{aligned}
$$

The control process can also be represented by the following diagram (Fig. 3):

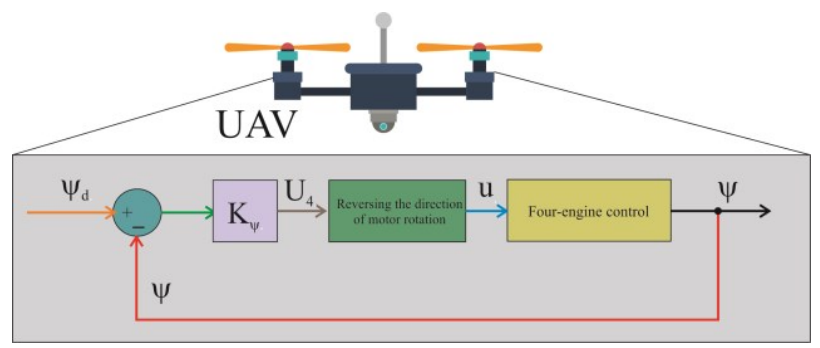

Fig. 3. The control loop of the deviation angle $\psi$

\subsubsection{Tilting and inclination control}

Because the 4-rotor UAV system is symmetrical, it can be assumed that the control of tilt and inclination at small angles is independent for each direction. In comparison to the deviation control, in this case a controller with a higher connection capacity is required, because the control in these directions is additionally directly related to the lateral acceleration in the $\mathrm{x}$ and $\mathrm{y}$ directions.

The tilt control is described in the following formula and diagram (Fig. 4):

$$
\begin{aligned}
U_{2}=K_{\phi_{P}}\left(\phi-\phi_{d}\right) & -K_{\phi_{I}} \int\left(\phi-\phi_{d}\right) \delta t \\
& -K_{\phi_{D}}\left(\dot{\phi}-\dot{\phi}_{d}\right)
\end{aligned}
$$

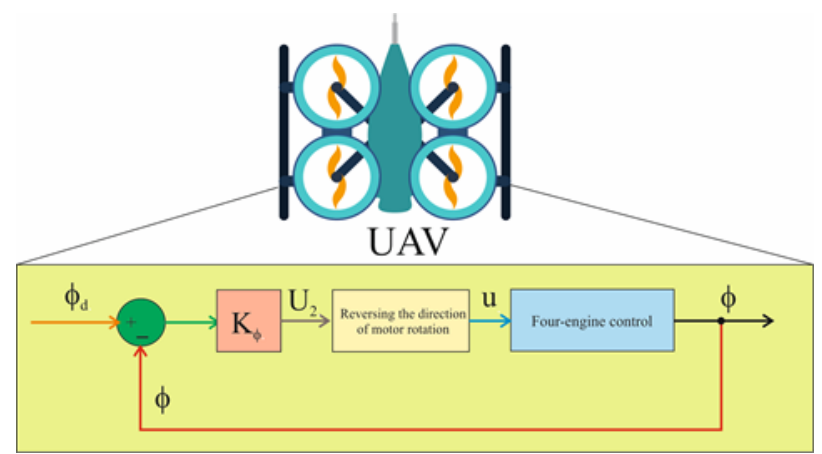

Fig. 4. Controller controlling the tilting angle Analogously for inclination control:

$$
\begin{gathered}
U_{3}=-K_{\theta_{P}}\left(\theta-\theta_{d}\right)-K_{\theta_{I}} \int\left(\theta-\theta_{d}\right) \delta t \\
-K_{\theta_{D}}\left(\dot{\theta}-\dot{\theta}_{d}\right)
\end{gathered}
$$

\subsubsection{Controller disposed in a horizontal position}

The position in the horizontal plane (described by the $x$ and $y$ axes) of the 4-rotor UAV is controlled by a continuous modification of the tilt and inclination. The total thrust creates the lift force that is approximately equal to the force of gravity $g$ in the direction perpendicular to the surface of the rotors. The low tilt angle $\phi$ therefore causes lateral acceleration of $y \approx \phi g$. In turn, the built-in controller is used to correct the UAV position in this way, as shown in Fig. 5.

Control in the $x$ and $y$ directions are shown in the following equations:

$$
\begin{aligned}
U_{x}=-K_{x_{p}}(x- & \left.x_{d}\right) \\
& -K_{x_{I}} \int\left(x-x_{d}\right) \delta t \\
& -K_{x_{D}}\left(\dot{x}-\dot{x}_{d}\right)
\end{aligned}
$$

$$
\begin{aligned}
U_{y}=-K_{y_{p}}(y- & \left.y_{d}\right) \\
& -K_{y_{I}} \int\left(y-y_{d}\right) \delta t \\
& -K_{y_{D}}\left(\dot{y}-\dot{y}_{d}\right)
\end{aligned}
$$

Equations corrected for the deviation angle for the designed tilt and inclination angles are as follows:

$$
\begin{gathered}
\phi_{d}=-\sin (\psi) U_{x}+\cos (\psi) U_{y} \\
\theta_{d}=\cos (\psi) U_{x}+\sin (\psi) U_{y}
\end{gathered}
$$

Fig. 5 shows the diagram of the $y$ position controller:

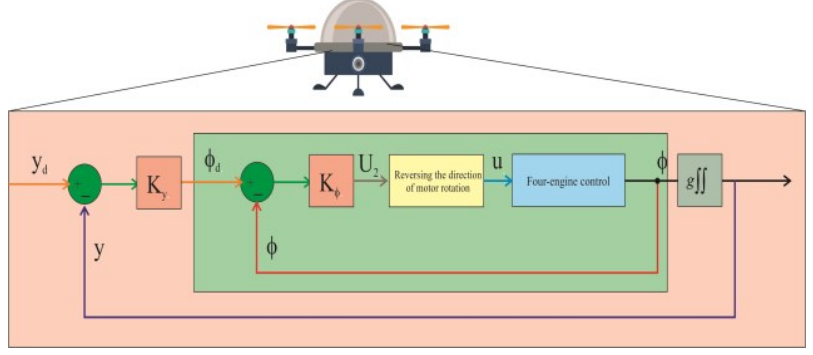

Fig. 5. Horizontal position $y$ calculated by the controller, for simplicity it is assumed that $\psi \approx 0$

\subsubsection{Controller disposed in a vertical position z}

When maneuvering above the influence of the earth effect, the output power of control $U_{l}$ is approximately proportional to the vertical acceleration relative to the structural reference system. However, to maintain a constant height, a large $U_{I}$ value is necessary to counteract the force of gravity [28], [29], [30].

In addition, in the case of classical control, an additional controller is added, which is responsible for stabilizing traffic in the $\mathrm{z}$ direction. The principle of operation can be described by the equation: 


$$
\begin{gathered}
r_{1}=-K_{z_{p}}\left(z-z_{d}\right) \\
-K_{z_{I}} \int\left(z-z_{d}\right) \delta t \\
-K_{z_{D}}\left(\dot{z}-\dot{z}_{d}\right) \\
U_{1}=\frac{r_{1}+m g}{\cos \phi \cos \theta}
\end{gathered}
$$

The diagram describing the operation of the vertical position controller $\mathrm{z}$ is described in Figure 6:

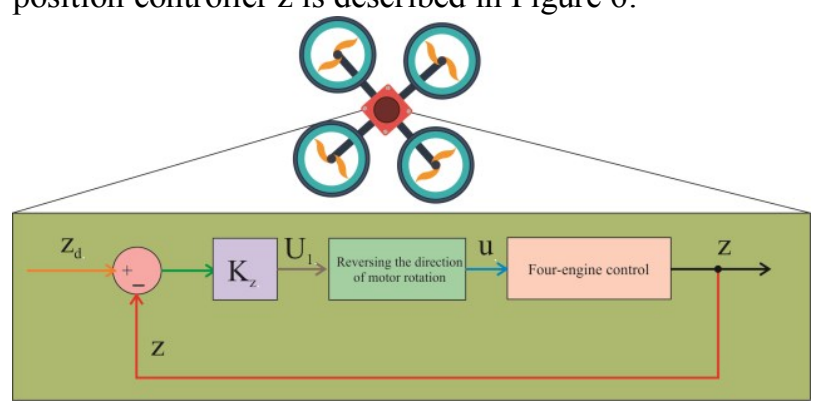

Fig. 6. Vertical position controller $z$

\subsubsection{Tracking the points}

The diagram describing the operation of the vertical co-ordination points determine the point in the space where the 4-rotor UAV should fly (i.e. $x_{d}, y_{d}, z_{d}$ ). In the case of hovering, only one point is needed, when a flight is made on a specific route, points and a time marker td must be created. There are two ways to do this: using discrete coordinate points or generating points via interpolation.

The second option is preferred, because in the case of entering incorrect information into the controller, its response is characterized by a smoother response than in the case of the first solution. In addition, the use of discrete points can cause system instability.

\section{Research results of 4-rotor UAV in the field of control}

Using the model described in the previous chapter, the discussed controller was tested in simulation in the Matlab/Simulink program. Using the parameters given in Table 1, the 4-rotor UAV is characterized by a stable hover. The results of the simulation are depicted in Figure 7.

The next figures (Figs. 8-9) show the results of the flight using the generation of coordination points by interpolation.

Table 1. Vertical position controller $z$

\begin{tabular}{|c|c|c|c|}
\hline Type of traffic & P & I & D \\
\hline Tilting & 0.9 & 0.3 & 0.2 \\
\hline Inclination & 0.9 & 0.3 & 0.2 \\
\hline Deviation & 0.06 & 0.01 & 0.02 \\
\hline
\end{tabular}

\begin{tabular}{|c|c|c|c|}
\hline $\mathrm{x}$ & -0.09 & 0 & -0.1 \\
\hline $\mathrm{y}$ & 0.09 & 0 & 0.1 \\
\hline $\mathrm{z}$ & 8 & 0.5 & 10 \\
\hline
\end{tabular}
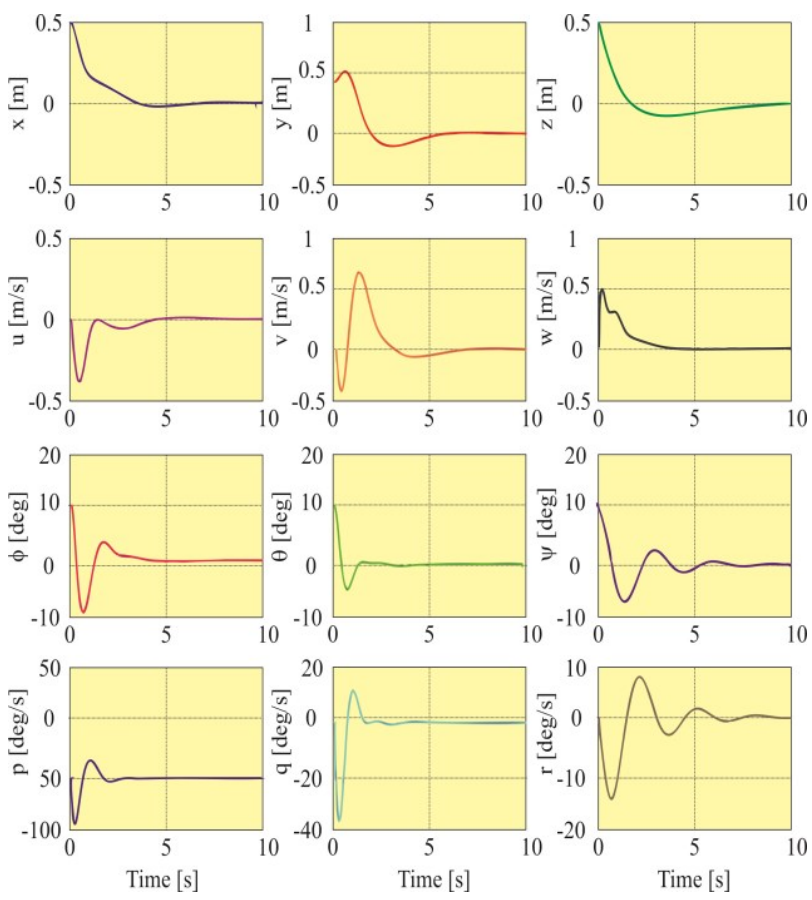

Fig. 7. Stability of the system in the hover. Initial parameters: $10^{\circ}$ for deviation, tilt and inclination, and $0.5 \mathrm{~m}$ for position $\mathrm{x}$, y, z.
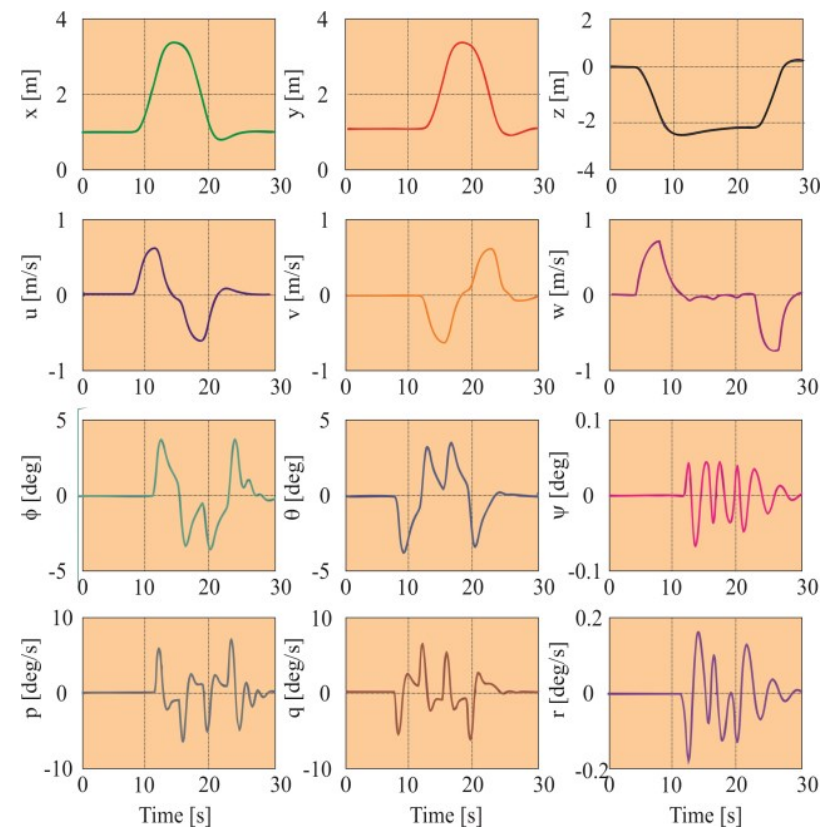

Fig. 8. Flight simulation by points created by interpolation Equations corrected for the deviation angle for the 


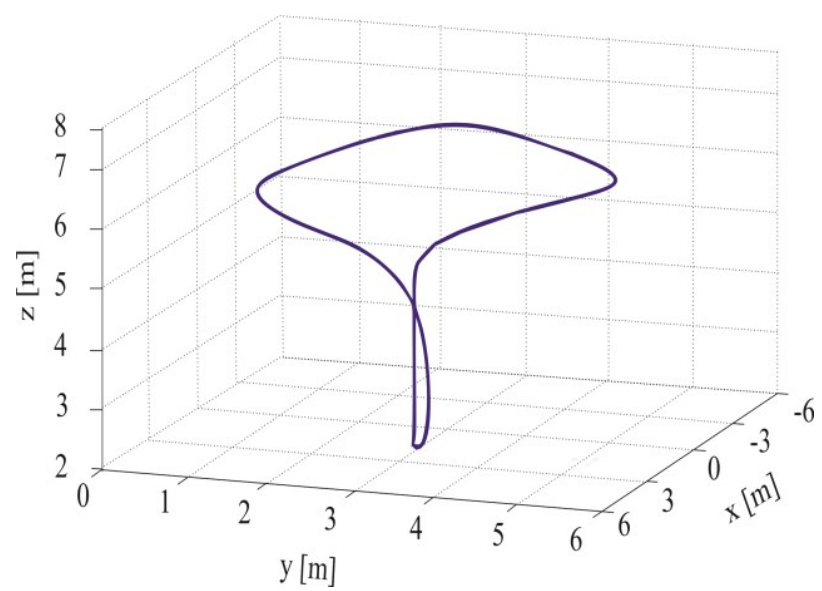

Fig. 9. Flight simulation by points created by interpolation

\section{Conclusions}

The selected type of control, i.e. classic control for each axis with an additional built-in controller responsible for maintaining the position, is able to ensure the stability of the 4-rotor UAV and enables flight on predetermined points in space.

Simulation of the use of control theory by means of the extended Kalman filter using infrared sensors shows that such a filter is able to correctly estimate the position of the object, hence it further improves the estimation of tilt angles, inclination and deviation of the 4-rotor UAV.

Additionally, when the polarization values of the accelerometers and gyroscopes decrease, the filter is still able to track the position of the device. In addition, this filter retains its observability even if there are not 6 infrared sensors in use, but five or four. Using three sensors, the filter loses observability, which results in the fact that estimation of the 4-rotor UAV position becomes impossible.

\section{References}

1. S. Bouabdallah, A. Noth, and R. Siegwart, "PID vs LQ Control Techniques Applied to an Indoor Micro Quadrotor," IEEE/RSJ International Conference on Intelligent Robots and Systems, vol. 3, pp. 24512456, 2004

2. I. C. Dikmen, A. Arisoy, and H. Temelta, "Attitude control of a quadrotor," 4th International Conference on Recent Advances in Space Technologies, pp. 722-727, 2009

3. G. M. Hoffmann, H. Huang, S. L. Waslander, and C.J. Tomlin, "Quadrotor helicopter flight dynamics and control: Theory and experiment," Proceedings of the AIAA Guidance, Navigation and Control Conference and Exhibit, Aug. 2007

4. T. Brcsciani, "Modelling, Identification and Control of a Quadrotor Helicopter," Master's thesis, Lund University, Sweden, 2008

5. D. Mcllingcr, Q. Lindsey, M. Shomin, and V. Kumar, "Design, modeling, estimation and control for aerial grasping and manipulation," in
Intelligent Robots and Systems (IROS), 2011 IEEE/RSJ International Conference on, pages 2668-2673, Sept. 2011

6. M. Huang, B. Xian, C. Diao, K. Yang, and Y. Feng, "Adaptive tracking control of underactuated quadrotor unmanned aerial vehicles via backstepping," in American Control Conference (ACC), 2010, pp. 2076-2081, 2010

7. T. Madani, and A. Benallegue, "Backstepping control for a quadrotor helicopter," IEEE/RSJ International Conference on Intelligent Robots and Systems, pp. 3255-3260, 2006

8. B. T. Whitehead, and S. R. Bieniawski. "Model Reference Adaptive Control of a Quadrotor UAV," In Guidance Navigation and Control Conference 2010, Toronto, Ontario, Canada, A1AA, 2010

9. P. Castillo, R. Lozano, and A. Dzul, "Stabilisation of a mini rotorcraft with four rotors, " IEEE Control Systems Magazine, pp. 45-55, Dec. 2005

10. I. Moir, A. Seabridge, "Design and Development of Aircraft Systems," Second Edition, John Wiley \& Sons, Ltd., 2013

11. L. Setlak, R. Kowalik, "Mathematical model and simulation of selected components of the EPS of the aircraft, providing the operation of on-board electrical equipment and systems in accordance with MEA/AEA concept," IEEE, pp. 1-6, PAEE 2017

12. J. Escareno, C. Salazar-Cruz, and R. Lozano, "Embedded control of a four-rotor UAV," American Control Conference, vol. 4, no. 11, pp. 3936-3941, 2006

13. D. Oldziej, Z. Gosiewski, "Modelling of Dynamic and Control of Six-Rotor Autonomous Unmanned Aerial Vehicle," Mechatronic Systems and Materials IV, Book Series: Solid State Phenomena, Vol. 198, pages: $220-225,2013$

14. H. Huang, G. M. Hoffmann, S. L. Waslander, and C. J. Tomlin, "Aerodynamics and control of autonomous quadrotor helicopters in aggressive maneuvering," IEEE International Conference on Robotics and Automation, pp. 3277-3282, 2009

15. I.D. Cowling, O.A. Yakimcnko, J.F. Whidbornc, and A.K. Cooke, "A prototype of an autonomous controller for a quadrotor UAV," in European Control Conference, pp. 1-8, 2007

16. J.J. Craig, P. Hsu, and. S.S. Sastry, "Adaptive control of mechanical manipulators," The International Journal of Robotics Research, 6 (2): 16,1987

17. Z. Zuo, "Trajectory tracking control design with command filtered compensation for a quadrotor," IET Control Theory and Applications, vol. 4, no. 11, pp. 2343-2355, 2010

18. D. Lee, H. Jin Kim, and S. Sastry, "Feedback linearization vs. adaptive sliding mode control for a quadrotor helicopter," International Journal of 
Control, Automation and Systems, 7(3), pp. 419428, 2009

19. A. Das, K. Subbarao, and F. Lewis, "Dynamic inversion with zero-dynamics stabilisation for quadrotor control," Control Theory and Applications, IET, 3(3): 303-314, 2009

20. L. Setlak, R. Kowalik "Analysis, Mathematical Model and Simulation of Selected Components of Advanced Technological Solutions of HVDC Architecture of Modern Aircraft Compatible with the Concept of MEA/AEA," Lecture Notes in Engineering and Computer Science: Proceedings of The World Congress on Engineering and Computer Science, pp. 327-332, 2017

21. C. Kownacki, D. Oldziej, "Fixed-Wing UAVs Flock Control through Cohesion and Repulsion Behaviours Combined with a Leadership," International Journal of Advanced Robotic Systems, Vol. 13, No. 36, 2016

22. P. Pounds, R. Mahony, and P. Corke, "Modelling and control of a quadrotor robot," in Australasian conference on robotics and automation 2006, Auckland, NZ, 2006

23. W. Zeng, B. Xian, C. Diao, Q. Yin, H. Li, and Y. Yang, "Nonlinear adaptive regulation control of a quadrotor unmanned aerial vehicle," in Control Applications (CCA), 2011 IEEE International Conference on, pp. 133-138, 2011

24. R. Xu, and U. Ozguner, "Sliding mode control of a quadrotor helicopter," in Decision and Control, IEEE Conference on, pp. 4957-4962, 2006

25. K. M. Zemalache, L. Beji, and H. Marref, "Control of an under-actuated system: Application to a four rotors rotorcraft," IEEE International Conference on Robotic and Biomimetic, pp. 404-409, 2005

26. Tayebi Abdelhamid, and S. McGilvray, "Attitude stabilization of a four-rotor aerial robot," 43rd IEEE Conference on Decision and Control, vol. 2, pp. 1216-1221, 2005

27. S. Bouabdallah, and R. Siegwart, "Backstepping and Sliding-mode Techniques Applied to an Indoor Micro Quadrotor," in Conference on Robotics and Automation, pp. 2247-2252, 2005

28. M. Grzegorzewski, "Results of a Research Predicting the Position of an Aircraft during Approach and Landing using the Bessel Function," Journal of Theoretical and Applied Mechanics, Vol. 51, No. 4, pp. 915-926, 2013

29. D. Oldziej, W. Walendziuk, K. Mirek, "Identification of Gas Powered Motor Propulsion Group for Small Unmanned Aerial Vehicles," Photonics Applications in Astronomy, Communications, Industry, and High-Energy Physics Experiments, pages 1-8, 2016

30. B. Oszczak, M. Grzegorzewski, J. Cwiklak, "Solution for Inter-Satellite Linked Space-Time Network Using Reference and Transition Point Indicators," Proceedings of the 27th International
Technical Meeting of the Satellite Division of the Institute of Navigation (ION GNSS 2014), pp. 23632370, 2014 